\title{
The Propositional Theory of Closure
}

\author{
A. E. McCluskey, D. W. McIntyre, and W. S. Watson
}

\section{CONTENTS}

1. Introduction

2. The Propositional Language $\mathfrak{C l}$

3. Elementary Properties and the System $\mathfrak{C l}_{-}$

4. Types and the Adequacy of $\mathfrak{C l}$

5. Universals and Properties of Spaces

6. Further Directions

7. Programs

References
2000 AMS Subject Classification: Primary 54A05; Secondary 54D10, 54F65, 54G05

Keywords: Closure operator, formal system, extremally disconnected space
We study the simplest fragment of topological theory: those statements that can be expressed using one set variable, interior and closure operators, and inclusion. We introduce a formal system that is simple enough to be implemented on a computer and exhaustively studied and yet rich enough to be sound and complete for the fragment of theory under consideration. This fragment is rich enough to capture concepts such as regular open sets, extremal disconnectedness, partition topologies, and the nodec property.

\section{INTRODUCTION}

It is well known that the structure of a topological space may be characterized by either its closure operator $\mathrm{cl}(\cdot)$ or its interior operator int $(\cdot)$. Moreover, any operator $\operatorname{cl}(\cdot): \mathbb{P}(X) \rightarrow \mathbb{P}(X)$ such that for all $A, B \subseteq X$,

1. $\operatorname{cl}(\operatorname{cl}(A))=\operatorname{cl}(A)$,

2. $A \subseteq \operatorname{cl}(A)$,

3. $\operatorname{cl}(A \cup B)=\operatorname{cl}(A) \cup \operatorname{cl}(B)$,

4. $\operatorname{cl}(\varnothing)=\varnothing$,

is the closure operator for a topology on $X$, with a similar characterization for interior operators (see, for example, [Engelking 89, Proposition 1.2.7]).

In this paper we explore the strength of a somewhat restricted theory, in which we are allowed only one set variable and a closure and an interior operator, and a relation symbol for $\subseteq$. We shall see that even this restricted language enables us to discuss familiar topological concepts such as the notion of regular open sets and that of extremally disconnected spaces.

We will explore formal systems in this restricted language, using "production systems" in which we identify a set $S$ of well-formed formulas, a subset $A$ of $S$ of axioms, and a set of rules of inference; for $\Gamma \subseteq S$ and $\varphi \in S$ we write $\Gamma \vdash \varphi$ if there is a finite sequence $\left(\psi_{1}, \psi_{2}, \ldots, \psi_{n}\right)$ 
with $\psi_{n}=\varphi$ such that each $\psi_{i}$ is in $\Gamma$, is in $A$, or follows from earlier terms in the sequence by a rule of inference. Such a sequence is called a derivation. We will exploit the equivalence of such systems with approaches using consequence operators Con $: \mathbb{P}(S) \rightarrow \mathbb{P}(S)$, where $\operatorname{Con}(\Gamma)=\{\varphi \in S \mid \Gamma \vdash \varphi\}$ is the smallest superset of $\Gamma$ that contains $A$ and is closed under the rules of inference. We will also use the fact that if $\Gamma, \Omega \subseteq S$ and $\varphi \in S$ with $\Omega \vdash \varphi$ and $\Gamma \vdash \psi$ for all $\psi \in \Omega$, then $\Gamma \vdash \varphi$.

We will describe a production system $\mathfrak{C l}$ that is intended to capture the theory of our restricted language. We will examine this system, showing it to be sound and adequate. Because the system is so simple, it is amenable to implementation and testing on a computer: many of our conjectures were tested using scripts written in the language Perl, described in the last section.

\section{THE PROPOSITIONAL LANGUAGE $\mathfrak{C l}$}

We now introduce the language of the proposed theory:

Definition 2.1. A word is a term that can be built from a single variable $\mathrm{A}$ and the two unary operations $\mathrm{cl}$ and int .

Examples of words are $\mathrm{clint} A$ and $\mathrm{cl} c \mathrm{int} \mathrm{cl} \operatorname{int} \operatorname{int} \mathrm{A}$.

The semantic intention, of course, is that A represent a subset of a topological space and that $\mathrm{cl}$ and int represent the closure and interior operators $\operatorname{cl}(\cdot)$ and int $(\cdot)$ in that space.

Given a word $w$ and a subset $A$ of a topological space $X$, let $w_{A}$ denote the corresponding subset of $X$ : more formally, we have

1. $\mathrm{A}_{A}=A$;

2. $(\mathrm{cl} w)_{A}=\operatorname{cl}\left(w_{A}\right)$;

3. (int $w)_{A}=\operatorname{int}\left(w_{A}\right)$.

Using four properties that are true in any topological space, it is well known that there are only seven "different" words [Kuratowski 22].

Proposition 2.2. Let $X$ be a topological space, and let $A \subseteq X$. Then we have

1. $\operatorname{cl}(\operatorname{cl}(A))=\operatorname{cl}(A)$;

2. $\operatorname{int}(\operatorname{int}(A))=\operatorname{int}(A)$;

3. $\operatorname{cl}(\operatorname{int}(\operatorname{cl}(\operatorname{int}(A))))=\operatorname{cl}(\operatorname{int}(A))$;

4. $\operatorname{int}(\operatorname{cl}(\operatorname{int}(\operatorname{cl}(A))))=\operatorname{int}(\operatorname{cl}(A))$;
From this it follows that we may restrict ourselves to the following seven "reduced" words:

$A, \quad c l A, \quad \operatorname{int} A, \quad \operatorname{int} c l A, \quad c l i n t A, \quad c l i n t c l A, \quad \operatorname{int} c l i n t A ;$

that is, for every word $w$ there is a reduced word $w^{\prime}$ such that for every $X$ and every $A \subseteq X$, we have $w_{A}=\left(w^{\prime}\right)_{A}$. We denote the above words by $v_{0}$ to $v_{6}$ respectively, and we denote $\left\{v_{0}, \ldots, v_{6}\right\}$ by $W$.

Definition 2.3. A property is a string of symbols of the form $w<v$, where $w$ and $v$ are reduced words. We denote the set of properties by $\mathcal{P}$.

Examples of properties include $\mathrm{A}<\mathrm{cl}$ int $\mathrm{A}$ and $\operatorname{int} \mathrm{A}<$ $c l \mathrm{~A}$. We denote the property $v_{i}<v_{j}$ by $(i j)$; for example, the property $\mathrm{A}<\mathrm{clint} \mathrm{A}$ will be denoted by (04).

Let $A$ be a subset of a space $X$, and let $P$ be the property $w<v$. We say that $A$ has property $P$ if $w_{A} \subseteq$ $v_{A}$.

Definition 2.4. We define the operations $c, i: W \rightarrow W$ as follows:

\begin{tabular}{|c|c|c|}
\hline$w$ & $c(w)$ & $i(w)$ \\
\hline $\bar{A}$ & $\mathrm{clA}$ & $\operatorname{int} A$ \\
\hline $\mathrm{cl} A$ & $\mathrm{cl} A$ & $\operatorname{int} \mathrm{cl} A$ \\
\hline $\operatorname{int} A$ & $\mathrm{clint} A$ & $\operatorname{int} A$ \\
\hline int $\mathrm{cl} A$ & $\mathrm{clint} \mathrm{cl} A$ & $\operatorname{int} \mathrm{cl} A$ \\
\hline $\mathrm{clint} A$ & $\mathrm{clint} A$ & $\operatorname{int} \mathrm{cl}$ int $\mathrm{A}$ \\
\hline $\mathrm{clint} \mathrm{cl} A$ & $\mathrm{clint} \mathrm{cl} A$ & $\operatorname{int} \mathrm{cl} A$ \\
\hline $\operatorname{int} \mathrm{cl}$ int $A$ & $\mathrm{clint} A$ & $\operatorname{int} \mathrm{clint} A$ \\
\hline
\end{tabular}

Syntactically, $c(w)$ is obtained by prepending $\mathrm{cl}$ to $w$ and then canceling according to the rules implied by Proposition 2.2, and $i(w)$ is obtained by prepending int and canceling. The semantic effect of the above operations is embodied in the following result.

Proposition 2.5. Let $w \in W$, and let $A \subseteq X$ for some topological space $X$. Then we have

1. $c(w)_{A}=\operatorname{cl}\left(w_{A}\right)$;

2. $i(w)_{A}=\operatorname{int}\left(w_{A}\right)$.

We may now introduce the system $\mathfrak{C}$. This system has as its well-formed formulas the properties $\mathcal{P}$. It has the following axioms:

A1 $\mathrm{A}<\mathrm{A}$.

A2 $w<c(w)$, for each $w \in W$. 
A3 $i(w)<w$, for each $w \in W$.

It has the following rules of inference:

Cl1 From $w<v$ and $v<u$ infer $w<u$.

Cl2 From $w<v$ infer $i(w)<i(v)$.

Cl3 From $w<v$ infer $c(w)<c(v)$.

Definition 2.6. Let $P_{1}, P_{2}, \ldots, P_{n}, Q \in \mathcal{P}$. We write $P_{1}, P_{2}, \ldots, P_{n} \vdash_{\mathfrak{C r}} Q$ if there is a derivation $R_{1}, R_{2}, \ldots, R_{k}$, where $R_{k}=Q$ and each $R_{i}$ is an axiom, is $P_{j}$ for some $j$, or follows from one or more of the earlier $R_{j}$ 's by one of the rules of inference. We write $P_{1}, P_{2}, \ldots, P_{n} \models Q$ if for every subset $A$ of a topological space $X$, if $A$ has property $P_{i}$ for $1 \leq i \leq n$, then $A$ has property $Q$.

As usual, our goal is to show that the syntactic notion and the semantic notion are equivalent.

Proposition 2.7. (Soundness of $\mathfrak{C l}$.) Let

$$
P_{1}, P_{2}, \ldots, P_{n}, Q \in \mathcal{P} \text {. }
$$

If $P_{1}, P_{2}, \ldots, P_{n} \vdash_{\mathfrak{C l}} Q$, then $P_{1}, P_{2}, \ldots, P_{n} \models Q$.

Proof: This follows easily by induction, since all the axioms and rules of inference are sound.

The proof that $\mathfrak{C l}$ is adequate will be given in Section 4. The system $\mathfrak{C l}$ is implemented in the script full_cons, described in Section 7.1. When this script is invoked with input a list of properties $P_{1}, P_{2}, \ldots, P_{n}$, its output is a list of all properties $Q$ such that $P_{1}, P_{2}, \ldots, P_{n} \vdash_{\mathfrak{C l}} Q$.

\section{ELEMENTARY PROPERTIES AND THE SYSTEM $\mathfrak{C l}_{-}$}

Although the set $\mathcal{P}$ of properties has only 49 elements, it is still too large to allow practical computations considering all subsets. We will identify a set $\mathcal{E}$ of "elementary" properties such that every property is equivalent to a (possibly empty) conjunction of elementary properties. First, we should define this notion of equivalence.

Definition 3.1. Let $P_{1}, P_{2}, \ldots, P_{n}, Q \in \mathcal{P}$. We write $Q \Leftrightarrow$ $P_{1} \wedge P_{2} \wedge \cdots \wedge P_{n}\left(\right.$ or $\left.Q \Leftrightarrow \bigwedge_{i=1}^{n} P_{i}\right)$ if

- for each $i, Q \vdash_{\mathfrak{C l}} P_{i}$; and

- $P_{1}, P_{2}, \ldots, P_{n} \vdash_{\mathfrak{C l}} Q$.
Note that it might have been more natural to define equivalence in terms of $\models$ rather than $\vdash_{\mathfrak{c} r}$ : when we have proved Theorem 4.5, we will see that the two notions of equivalence are equivalent. Note also that $Q \Leftrightarrow P_{1} \wedge P_{2} \wedge \cdots \wedge P_{n}$ if and only if $\operatorname{Con} \mathfrak{C r}(\{Q\})=$ $\operatorname{Con}_{\mathfrak{C r}}\left(\left\{P_{1}, P_{2}, \ldots, P_{n}\right\}\right)$.

We define $\mathcal{A}=\operatorname{Con}_{\mathfrak{C r}}(\varnothing)$. The properties in $\mathcal{A}$ may be identified using the script full_cons with no properties input: if we do so, we find that there are 23 such "tautological" properties. As a result, the script proper_cons was written: this script is identical to full_cons except that the 23 tautological properties are suppressed from the output. For $P \in \mathcal{P}$, we define the set of proper consequences of $P$ to be

$$
\begin{aligned}
& \operatorname{Con}_{\mathfrak{C r}}^{\prime}(P)=\operatorname{Con}_{\mathfrak{C l}}(P) \backslash \mathcal{A} \\
& \quad=\left\{Q \in \mathcal{P} \mid P \vdash_{\mathfrak{C l}} Q \text { and } Q \text { is not tautological }\right\} .
\end{aligned}
$$

Our first attempt at a definition of "elementary" is the following notion.

Definition 3.2. A property $P$ is absolutely elementary if there is no subset $S=\left\{Q_{1}, Q_{2}, \ldots, Q_{n}\right\} \subseteq \mathcal{P} \backslash\{P\}$ such that $P \Leftrightarrow \bigwedge_{i=1}^{n} Q_{i}$.

However, this fails to take into account pairs like (05) and (15), for which we have (05) $\Leftrightarrow(15)$, so neither property is absolutely elementary. However, $\operatorname{Con}_{\mathfrak{C l}}^{\prime}((05))=$ $\operatorname{Con}_{\mathfrak{C r}}^{\prime}((15))=\{(05),(15)\}$, so any set of elementary properties must include one or the other of these two.

Definition 3.3. We order $\mathcal{P}$ lexicographically, so that $(i j) \preccurlyeq\left(i^{\prime} j^{\prime}\right)$ if $i<i^{\prime}$ or $i=i^{\prime}$ and $j \leq j^{\prime}$. Then a property $P$ is canonical if $P \preccurlyeq Q$ for every $Q$ with $P \Leftrightarrow Q$.

To determine the canonical properties, we use the script canonical, described in Section 7.2. From this script we find that there are 21 canonical properties: we denote the set of canonical properties by $\mathcal{C}$. We have

$$
\begin{aligned}
& \mathcal{C}=\{(00),(02),(03),(04),(05),(06),(10),(12),(13), \\
& \\
&(16),(30),(34),(40),(42),(43),(46),(50),(52), \\
&(53),(56),(60)\} .
\end{aligned}
$$

We can now give a more suitable definition of elementary.

Definition 3.4. A property $P$ is elementary if $P \in \mathcal{C}$ and there is no subset $S=\left\{Q_{1}, Q_{2}, \ldots, Q_{n}\right\} \subseteq \mathcal{C} \backslash\{P\}$ such that $P \Leftrightarrow \bigwedge_{i=1}^{n} Q_{i}$. 
The elementary properties $\mathcal{E}$ can be found using the script find_elementaries described in Section 7.4. They are

(02) $A<\operatorname{int} A$

(03) $\mathrm{A}<\operatorname{int} \mathrm{cl} A$

(05) $\mathrm{A}<\mathrm{clint} \mathrm{cl} \mathrm{A}$

(10) $\mathrm{cl} \mathrm{A}<\mathrm{A}$

(34) $\operatorname{int} \mathrm{cl} \mathrm{A}<\mathrm{clint} \mathrm{A}$

(40) $\operatorname{clint} \mathrm{A}<\mathrm{A}$

(43) $\operatorname{clint} A<\operatorname{int} c l A$

(46) $\operatorname{clint} A<\operatorname{int} c l i n t A$

(53) $\operatorname{clint} \mathrm{cl} A<\operatorname{int} \mathrm{cl} A$

(60) $\operatorname{int} c \mathrm{int} A<A$

Using the script check_elementary with this list of elementary properties, we can verify that each property is indeed (either tautological or) equivalent to a conjunction of properties in $\mathcal{E}$.

We note that many of these elementary properties have been studied in the literature; see, for example, [Cao et al. 01] and [Isomichi 71].

Definition 3.5. Let $X$ be a topological space. A subset $A$ of $X$ is called:

- regular open if $A=\operatorname{int}(\operatorname{cl}(A))$;

- regular closed if $A=\operatorname{cl}(\operatorname{int}(A))$;

- $\alpha$-closed if $\operatorname{cl}(\operatorname{int}(\operatorname{cl}(A))) \subseteq A$;

- semiclosed (or supercondensed) if $\operatorname{int}(\operatorname{cl}(A)) \subseteq A$;

- preclosed if $\operatorname{cl}(\operatorname{int}(A)) \subseteq A$;

- $\beta$-closed if $\operatorname{int}(\operatorname{cl}(\operatorname{int}(A))) \subseteq A$;

- $\alpha$-open if $X \backslash A$ is $\alpha$-closed, or equivalently, if $A \subseteq$ $\operatorname{int}(\operatorname{cl}(\operatorname{int}(A)))$;

- semiopen (or subcondensed) if $X \backslash A$ is semiclosed, or equivalently, if $A \subseteq \operatorname{cl}(\operatorname{int}(A))$;

- preopen if $X \backslash A$ is preclosed, or equivalently, if $A \subseteq$ $\operatorname{int}(\operatorname{cl}(A))$;

- $\beta$-open if $X \backslash A$ is $\beta$-closed, or equivalently, if $A \subseteq$ $\operatorname{cl}(\operatorname{int}(\operatorname{cl}(A)))$.
Thus the elementary property (02) is "open," (10) is "closed," (03) is "preopen," (40) is "preclosed," (05) is " $\beta$-open," and (60) is " $\beta$-closed." The remaining properties in Definition 3.5 are conjunctions of elementary properties and are identified in Table 1.

For $S \subseteq \mathcal{P}$, we define the "elementary consequences" of $S$ to be $e c(S)=\operatorname{Con}_{\mathfrak{C r}}(S) \cap \mathcal{E}$.

We will now introduce the system $\mathfrak{C l}_{-}$, which is essentially $\mathfrak{C l}$ restricted to $\mathcal{E}$. The system has $\mathcal{E}$ as its set of well-formed formulas. It has no axioms, and has as its rules of inference the following:

Clm1 From (02) infer (03), i.e., from $A<\operatorname{int~} A$ infer $A<\operatorname{int} c l A$.

Clm2 From (03) infer (05), i.e., from $\mathrm{A}<\operatorname{int} \mathrm{cl} \mathrm{A}$ infer $\mathrm{A}<\mathrm{cl}$ int $\mathrm{cl} \mathrm{A}$.

Clm3 From (02) infer (34), i.e., from $\mathrm{A}<\operatorname{int} \mathrm{A}$ infer $\operatorname{int} \mathrm{cl} A<\operatorname{clint} \mathrm{A}$.

Clm4 From (10) infer (34), i.e., from $\mathrm{cl} A<\mathrm{A}$ infer $\operatorname{int} \mathrm{cl} A<\operatorname{cl}$ int $\mathrm{A}$.

Clm5 From (10) infer (40), i.e., from cl $\mathrm{A}<\mathrm{A}$ infer clint $A<A$.

Clm6 From (40) infer (60), i.e., from cl int $\mathrm{A}<\mathrm{A}$ infer int $c l$ int $A<A$.

Clm7 From (53) infer (43), i.e., from $\mathrm{cl}$ int $\mathrm{cl} A<\operatorname{int} \mathrm{cl} A$ infer $c l$ int $A<\operatorname{int} c l A$.

Clm8 From (46) infer (43), i.e., from cl int A $<\operatorname{int} c l$ int A infer $c l$ int $A<\operatorname{int} c l A$.

Clm9 From (34) and (43) infer (46), i.e., from int cl A $<$ $c l i n t A$ and $\operatorname{clint} A<\operatorname{intcl} A \operatorname{infer} \operatorname{clint} A<$ int $c l$ int $A$.

Clm10 From (34) and (43) infer (53), i.e., from int cl A < $c l i n t A$ and $c l i n t A<\operatorname{int} c l A \operatorname{infer} c l i n t c l A<\operatorname{int} c l A$.

Clm11 From (05) and (53) infer (03), i.e., from $\mathrm{A}<$ $\mathrm{clint} c l \mathrm{~A}$ and $\mathrm{clint} \mathrm{cl} A<\operatorname{int} \mathrm{cl} A \operatorname{infer} \mathrm{A}<\operatorname{int} \mathrm{cl} A$.

Clm12 From (46) and (60) infer (40), i.e., from cl int $A<$ $\operatorname{int} c l \operatorname{int} A$ and $\operatorname{int} c l i n t A<A$ infer $c l i n t A<A$.

Clm13 From (03) and (40) infer (43), i.e., from $\mathrm{A}<$ $\operatorname{int} \mathrm{cl} A$ and $\mathrm{clint} A<A$ infer $c l \operatorname{int} A<\operatorname{int} \mathrm{cl} A$.

Clm14 From (03) and (34) and (60) infer (02), i.e., from $A<\operatorname{int} c l A$ and $\operatorname{int} c l A<\operatorname{cl} A$ t $A$ and $\operatorname{int} c l i n t A<A$ infer $A<\operatorname{int} A$. 
Clm15 From (05) and (34) and (40) infer (10), i.e., from $A<c l i n t c l A$ and int $c l A<c l i n t A$ and $c l i n t A<A$ infer $\mathrm{cl} A<\mathrm{A}$.

Proposition 3.6. For all $P_{1}, P_{2}, \ldots, P_{n}, Q \in \mathcal{E}$ we have $P_{1}, P_{2}, \ldots, P_{n} \vdash_{\mathfrak{C l}} Q$ if and only if $P_{1}, P_{2}, \ldots, P_{n} \vdash_{\mathfrak{C l}_{-}} Q$.

Proof: For each subset $S=\left\{P_{1}, P_{2}, \ldots, P_{n}\right\}$ of $\mathcal{E}$ we can find all the consequences of $S$ in $\mathfrak{C l}$ and in $\mathfrak{C l}_{-}$. The result we are proving is that $e c(S)=\operatorname{Con}_{\mathfrak{C l}}(S) \cap \mathcal{E}=\operatorname{Con}_{\mathfrak{C l}_{-}}(S)$ for every $S \subseteq \mathcal{E}$.

The script cl-minus described in Section 7.5 calculates $\operatorname{ec}(S)$ and $\mathrm{Con}_{\mathfrak{C r}_{-}}(S)$ for each $S \subseteq E$ and verifies that they are equal, as required.

In fact, the list $\mathbf{C l m 1 - C l m 1 5}$ was found using this script. The list of rules of inference that we initially conjectured to be sufficient had 19 rules, but was not strong enough to derive all consequences. With the use of this script, we were able to find the appropriate strengthening of two of the rules in our original list, and also to discover that four of the rules in our original list could be derived from the remaining rules.

\section{TYPES AND THE ADEQUACY OF $\mathfrak{C l}$}

The goal of this section is to show that $\mathfrak{C l}$ is adequate, in other words, that if $P_{1}, P_{2}, \ldots, P_{n} \models Q$, then $P_{1}, P_{2}, \ldots, P_{n} \vdash_{\mathfrak{c r}} Q$. In fact, we will show the contrapositive: if $P_{1}, P_{2}, \ldots, P_{n} \forall_{\mathfrak{C r}} Q$, then $P_{1}, P_{2}, \ldots, P_{n} \forall Q$. Furthermore, by the results in Section 3 we may restrict our attention to the case that $P_{1}, P_{2}, \ldots, P_{n}, Q \in \mathcal{E}$ and $P_{1}, P_{2}, \ldots, P_{n} \nvdash_{\mathfrak{C l}_{-}} Q$.

Definition 4.1. Let $A$ be a subset of a topological space $X$. The type of $A$, type $(A)$, is the set of $P \in \mathcal{E}$ such that $A$ has property $P$.

A type is a subset $T$ of $\mathcal{E}$ such that $T=\operatorname{type}(A)$ for some subset $A$ of some topological space $X$.

Lemma 4.2. If $S$ is a type, then $S$ is closed under $\vdash_{\mathfrak{C r}_{-}}$ (in other words, if $Q \in \mathcal{E}$ and $S \vdash_{\mathfrak{C l}_{-}} Q$, then $Q \in S$ ).

Proof: This follows from the fact that all 15 rules of inference are sound.

Lemma 4.3. There are exactly 49 subsets of $\mathcal{E}$ that are closed under $\vdash_{\mathfrak{C l}_{-}}$.

Proof: For each of the 1024 subsets $S$ of $\mathcal{E}$ it is a simple matter to determine whether $S$ is closed under each of the 15 rules of inference. The script types described in Section 7.6 does this. Its output is a list of 49 subsets, which are given in Table 1 . In what follows, we will refer to these sets as Type 0 to Type 48 .

We will now show that these 49 potential types are indeed all types. To do this we will give examples of topological spaces $X_{i}$ and subsets $S_{i}$ for $0 \leq i \leq 48$ such that $S_{i}$ has type $i$. The spaces $X_{i}$ will be finite. We will actually define the topology on $X_{i}$ by specifying a preorder on $X_{i}$, in other words, a relation that is reflexive and transitive (but not necessarily antisymmetric). For each such preorder $\leq$ there is an associated topology given by declaring that $U \subseteq X_{i}$ is open if for every $x \in U$ and every $y$ with $x \leq y$ we have $y \in U$. Equivalently, for each topology we may define a preorder $\leq$ by declaring that

$$
x \leq y \text { if } x \in \operatorname{cl}(\{y\})
$$

This preorder is called the specialization order for the topology.

Example 4.4. For each $i$ with $0 \leq i \leq 48$ we will construct a preorder on the ten-element set $X_{i}=\{a, b, \ldots, j\}$ and find a subset $S_{i}$ of $X_{i}$ that has Type $i$ in the corresponding topology. These examples were found using the script find_types described in Section 7.6.

The examples are listed in Table 2. For example, the row "Type $16|\{d, f\}| d i, g f, i d$ " means that the subset $\{d, f\}$ of $X_{16}$, which has topology with specialization order generated by $d<i, g<f, i<d$, has Type 16 $(\{(03),(05),(43),(46),(53)\})$.

Theorem 4.5. (Adequacy of $\mathfrak{C l}$.) For any $P_{1}, P_{2}, \ldots, P_{n}$, $Q \in \mathcal{P}$, if $P_{1}, P_{2}, \ldots, P_{n}=Q$, then $P_{1}, P_{2}, \ldots, P_{n} \vdash_{\mathfrak{C r}} Q$.

Proof: Suppose that $P_{1}, P_{2}, \ldots, P_{n} \forall_{\mathfrak{c r}} Q$. For each $i$, choose $R_{i, 1}, \ldots, R_{i, k_{i}} \in \mathcal{E}$ with $P_{i} \Leftrightarrow \bigwedge_{j=1}^{k_{i}} R_{i, j}$. Choose $S_{1}, \ldots, S_{k} \in \mathcal{E}$ with $Q \Leftrightarrow \bigwedge_{j=1}^{k} S_{j}$. Put $\Gamma=\left\{R_{i, j} \mid 1 \leq\right.$ $\left.i \leq n, 1 \leq j \leq k_{i}\right\}$.

We claim that there is some $j_{0}$ such that $\Gamma \forall_{\mathfrak{C} \mathfrak{r}_{-}} S_{j_{0}}$. If this were not true, then we would have $\Gamma \vdash_{\mathfrak{C r}} S_{j}$ for all $j$ (by Proposition 3.6). Hence, since $S_{1}, \ldots, S_{k} \vdash_{\mathfrak{C r}} Q$, we would have $\Gamma \vdash_{\mathfrak{c r}} Q$. We also have $P_{i} \vdash_{\mathfrak{c l}} R_{i, j}$ for all $i, j$, so $P_{1}, \ldots, P_{n} \vdash_{\mathfrak{c l}} R$ for all $R \in \Gamma$. Thus $P_{1}, \ldots, P_{n} \vdash_{\mathfrak{C r}} Q$, contradicting our assumption. 


\begin{tabular}{|c|c|c|}
\hline Type 0 & $\varnothing$ & \\
\hline Type 1 & $\{(05)\}$ & $\beta$-open \\
\hline Type 2 & $\{(03),(05)\}$ & preopen \\
\hline Type 3 & $\{(34)\}$ & \\
\hline Type 4 & $\{(05),(34)\}$ & semi-open \\
\hline Type 5 & $\{(03),(05),(34)\}$ & $\alpha$-open \\
\hline Type 6 & $\{(02),(03),(05),(34)\}$ & open \\
\hline Type 7 & $\{(43)\}$ & \\
\hline Type 8 & $\{(05),(43)\}$ & \\
\hline Type 9 & $\{(03),(05),(43)\}$ & \\
\hline Type 10 & $\{(43),(46)\}$ & \\
\hline Type 11 & $\{(05),(43),(46)\}$ & \\
\hline Type 12 & $\{(03),(05),(43),(46)\}$ & \\
\hline Type 13 & $\{(43),(53)\}$ & \\
\hline Type 14 & $\{(03),(05),(43),(53)\}$ & \\
\hline Type 15 & $\{(43),(46),(53)\}$ & \\
\hline Type 16 & $\{(03),(05),(43),(46),(53)\}$ & \\
\hline Type 17 & $\{(34),(43),(46),(53)\}$ & \\
\hline Type 18 & $\{(03),(05),(34),(43),(46),(53)\}$ & \\
\hline Type 19 & $\{(02),(03),(05),(34),(43),(46),(53)\}$ & \\
\hline Type 20 & $\{(60)\}$ & $\beta$-closed \\
\hline Type 21 & $\{(05),(60)\}$ & \\
\hline Type 22 & $\{(03),(05),(60)\}$ & \\
\hline Type 23 & $\{(34),(60)\}$ & semiclosed \\
\hline Type 24 & $\{(05),(34),(60)\}$ & condensed \\
\hline Type 25 & $\{(02),(03),(05),(34),(60)\}$ & regular open \\
\hline Type 26 & $\{(40),(60)\}$ & preclosed \\
\hline Type 27 & $\{(05),(40),(60)\}$ & \\
\hline Type 28 & $\{(34),(40),(60)\}$ & $\alpha$-closed \\
\hline Type 29 & $\{(10),(34),(40),(60)\}$ & closed \\
\hline Type 30 & $\{(05),(10),(34),(40),(60)\}$ & regular closed \\
\hline Type 31 & $\{(43),(60)\}$ & \\
\hline Type 32 & $\{(05),(43),(60)\}$ & \\
\hline Type 33 & $\{(03),(05),(43),(60)\}$ & \\
\hline Type 34 & $\{(40),(43),(60)\}$ & \\
\hline Type 35 & $\{(05),(40),(43),(60)\}$ & \\
\hline Type 36 & $\{(03),(05),(40),(43),(60)\}$ & \\
\hline Type 37 & $\{(40),(43),(46),(60)\}$ & \\
\hline Type 38 & $\{(05),(40),(43),(46),(60)\}$ & \\
\hline Type 39 & $\{(03),(05),(40),(43),(46),(60)\}$ & \\
\hline Type 40 & $\{(43),(53),(60)\}$ & \\
\hline Type 41 & $\{(03),(05),(43),(53),(60)\}$ & \\
\hline Type 42 & $\{(40),(43),(53),(60)\}$ & \\
\hline Type 43 & $\{(03),(05),(40),(43),(53),(60)\}$ & \\
\hline Type 44 & $\{(40),(43),(46),(53),(60)\}$ & \\
\hline Type 45 & $\{(03),(05),(40),(43),(46),(53),(60)\}$ & \\
\hline Type 46 & $\{(34),(40),(43),(46),(53),(60)\}$ & \\
\hline Type 47 & $\{(10),(34),(40),(43),(46),(53),(60)\}$ & \\
\hline Type 48 & $\{(02),(03),(05),(10),(34),(40),(43),(46),(53),(60)\}$ & \\
\hline
\end{tabular}

TABLE 1. The 49 types of subsets.

Now let $\Pi$ be the smallest subset of $\mathcal{E}$ that contains $\Gamma$ and is closed under $\vdash_{\mathfrak{C l}_{-}}$. Then $S_{j_{0}} \notin \Pi$. By Lemmas 4.2 and 4.3 and Example 4.4, $\Pi$ is a type, so there is a subset $A$ of a space $X$ such that type $(A)=\Pi$. Then $A$ has property $R_{i, j}$ for all $i$ and $j$, and so $A$ has property $P_{i}$ for each $i$. On the other hand, since $A$ does not have property $S_{j_{0}}, A$ does not have property $Q$. Thus $P_{1}, P_{2}, \ldots, P_{n} \not \models Q$.

\section{UNIVERSALS AND PROPERTIES OF SPACES}

Up to this point we have considered properties of a given subset of a topological space $X$. We turn now to the question of properties of the space $X$ that can be characterized in our system. In the first instance we consider "universals," which are properties of spaces that may be defined by insisting that all subsets have certain proper- 


\begin{tabular}{|l|l|l|}
\hline Type & Subset & Order \\
\hline Type 0 & $\{a, g, i\}$ & $a h, b d, b j, c h, g f, h a, j i$ \\
Type 1 & $\{a, b, i\}$ & $a h, b d, b j, c h, g f, h a, j i$ \\
Type 2 & $\{a, i\}$ & $a h, b d, b j, c h, g f, h a, j i$ \\
Type 3 & $\{a, d\}$ & $b a, d i, f d, f h, j a, j h$ \\
Type 4 & $\{f, i\}$ & $b a, d i, f d, f h, j a, j h$ \\
Type 5 & $\{g, h\}$ & $a c, a h, b a, c i, d h, g d$ \\
Type 6 & $\{a\}$ & $b a, d i, f d, f h, j a, j h$ \\
Type 7 & $\{b, e, i\}$ & $a f, b h, c b, c e, d b, e g, f h, f i, h b, j i$ \\
Type 8 & $\{a, b, g\}$ & $a f, b h, c b, c e, d b, e g, f h, f i, h b, j i$ \\
Type 9 & $\{b, g\}$ & $a f, b h, c b, c e, d b, e g, f h, f i, h b, j i$ \\
Type 10 & $\{b, c, e\}$ & $b h, d c, e i, f b, f j, h b, i j$ \\
Type 11 & $\{c, f, g\}$ & $c e, c h, d g, f h, h f$ \\
Type 12 & $\{f, g\}$ & $c e, c h, d g, f h, h f$ \\
Type 13 & $\{b, d, j\}$ & $b h, d c, e i, f b, f j, h b, i j$ \\
Type 14 & $\{b, j\}$ & $b h, d c, e i, f b, f j, h b, i j$ \\
Type 15 & $\{a, b, c\}$ & $a e, b j, e a, i c$ \\
Type 16 & $\{d, f\}$ & $d i, g f, i d$ \\
Type 17 & $\{d, f\}$ & $f e, h d, j i$ \\
Type 18 & $\{d, h\}$ & $a e, a g, b d, h b$ \\
Type 19 & $\{d\}$ & $f e, h d, j i$ \\
Type 20 & $\{c, e, j\}$ & $b e, b f, c h, h c, j d$ \\
Type 21 & $\{b, c, g\}$ & $b h, c d, c h, h b, i c, j c, j g$ \\
Type 22 & $\{b, d\}$ & $b h, c d, c h, h b, i c, j c, j g$ \\
Type 23 & $\{b, h\}$ & $b a, d i, f d, f h, j a, j h$ \\
Type 24 & $\{f, h\}$ & $b a, d i, f d, f h, j a, j h$ \\
\hline
\end{tabular}

\begin{tabular}{|l|l|l|}
\hline Type & Subset & Order \\
\hline Type 25 & $\{h\}$ & $b a, d i, f d, f h, j a, j h$ \\
Type 26 & $\{b, c, e, j\}$ & $b e, b f, c h, h c, j d$ \\
Type 27 & $\{b, g, j\}$ & $b h, c d, c h, h b, i c, j c, j g$ \\
Type 28 & $\{a, b, e\}$ & $a e, a g, b d, h b$ \\
Type 29 & $\{a, e, h\}$ & $a e, a g, b d, h b$ \\
Type 30 & $\{a, e\}$ & $a e, a g, b d, h b$ \\
Type 31 & $\{a, b, f\}$ & $b h, c d, c i, d a, d c, d i, e h, e j, f i, i f, j f, j h$ \\
Type 32 & $\{a, e, g\}$ & $a h, c a, c g, e a, e j, f a, h f$ \\
Type 33 & $\{a, g\}$ & $a h, c a, c g, e a, e j, f a, h f$ \\
Type 34 & $\{b, c, e, h\}$ & $a c, b c, b e, c j, d h, d j, f c, f d, g c, g i, h i, j c$ \\
Type 35 & $\{a, c, e, g\}$ & $a h, c a, c g, e a, e j, f a, h f$ \\
Type 36 & $\{a, c, g\}$ & $a h, c a, c g, e a, e j, f a, h f$ \\
Type 37 & $\{d, f\}$ & $c e, c h, d g, f h, h f$ \\
Type 38 & $\{b, c\}$ & $b h, c d, c h, h b, i c, j c, j g$ \\
Type 39 & $\{b\}$ & $b h, c d, c h, h b, i c, j c, j g$ \\
Type 40 & $\{d, e, f\}$ & $c e, c h, d g, f h, h f$ \\
Type 41 & $\{e, f\}$ & $c e, c h, d g, f h, h f$ \\
Type 42 & $\{c, d, e, f\}$ & $c e, c h, d g, f h, h f$ \\
Type 43 & $\{c, e, f\}$ & $c e, c h, d g, f h, h f$ \\
Type 44 & $\{d, g\}$ & $d i, g f, i d$ \\
Type 45 & $\{d\}$ & $d i, g f, i d$ \\
Type 46 & $\{d\}$ & $b a, d i, f d, f h, j a, j h$ \\
Type 47 & $\{f\}$ & $f e, h d, j i$ \\
Type 48 & $\varnothing$ & $f e, h d, j i$ \\
\hline
\end{tabular}

TABLE 2. Subsets having all 49 types.

ties. In other words, these are spaces that may be characterized by a statement of the form $(\forall \mathrm{A}) \bigwedge_{i=1}^{n} P_{i}$, where for each $P_{i}, P_{i} \in \mathcal{E}$.

We first ask how many such universals there are. For example, $(\forall A)(c l A<A)$ is equivalent to $(\forall A)(A<\operatorname{int} A)$ : insisting that every subset be closed is the same as insisting that every subset be open.

Definition 5.1. Let $X$ be a topological space. The universal of $X, u(X)$, is the set of $P \in \mathcal{E}$ such that every subset of $X$ has property $P$, or equivalently, $u(X)=$ $\bigcap_{A \subseteq X} \operatorname{type}(A)$.

$\overline{\mathrm{A}}$ universal is a subset $S$ of $\mathcal{E}$ such that $S=u(X)$ for some topological space $X$.

To find the universals, we will extend $\mathfrak{C l}$ to a system $\mathfrak{U}$ : whereas $S \vdash_{\mathfrak{C l}} P$ was intended to mean, "if $A$ has all the properties in $S$, then $A$ has property $P, " S \vdash_{\mathfrak{U}} P$ is intended to mean, "if every subset of $X$ has all the properties in $S$, then every subset of $X$ has property $P$."

We first introduce three further operations on $W$.

Definition 5.2. We define the operations $d, C, I: W \rightarrow$ $W$ as follows:

\begin{tabular}{|c|c|c|c|}
\hline$w$ & $d(w)$ & $C(w)$ & $I(w)$ \\
\hline A & $A$ & $\mathrm{cl} A$ & $\operatorname{int} A$ \\
\hline $\mathrm{cl} A$ & $\operatorname{int} A$ & $\mathrm{cl} A$ & $\mathrm{clint} A$ \\
\hline $\operatorname{int} A$ & $\mathrm{cl} A$ & $\operatorname{int} \mathrm{cl} A$ & $\operatorname{int} A$ \\
\hline int $\mathrm{cl} A$ & $\mathrm{clint} A$ & $\operatorname{int} \mathrm{cl} A$ & $\operatorname{int} \mathrm{cl}$ int $A$ \\
\hline $\mathrm{clint} A$ & int $\mathrm{cl} A$ & $\mathrm{clint} \mathrm{cl} A$ & clint A \\
\hline $\mathrm{clint} \mathrm{cl} A$ & $\operatorname{int} \mathrm{cl}$ int $\mathrm{A}$ & $\mathrm{clint} \mathrm{cl} A$ & $\mathrm{clint} A$ \\
\hline int $\mathrm{cl}$ int $\mathrm{A}$ & $\mathrm{clint} \mathrm{cl} A$ & int $\mathrm{cl} A$ & $\operatorname{int} \mathrm{cl}$ int $\mathrm{A}$ \\
\hline
\end{tabular}

Syntactically, $d(w)$ is obtained by replacing each cl with int and vice versa, $C(w)$ is obtained by replacing A by $\mathrm{cl} A$ in $w$ and then canceling according to the rules implied by Proposition 2.2, and $I(w)$ is obtained by replacing $A$ by int $A$ and canceling.

The semantic effect of the above operations is embodied in the following result.

Proposition 5.3. Let $w \in W$, and let $A \subseteq X$ for some topological space $X$. Then we have

1. $d(w)_{A}=\left(w_{A^{\prime}}\right)^{\prime}$, where $B^{\prime}=X \backslash B$;

2. $C(w)_{A}=w_{\operatorname{cl}(A)}$;

3. $I(w)_{A}=w_{\operatorname{int}(A)}$. 


\begin{tabular}{|c|l|l|}
\hline & $U \cap \mathcal{E}_{\mathcal{U}}$ & $U$ \\
\hline quasiuniversal 0 & $\varnothing$ & $\varnothing$ \\
quasiuniversal 1 & $\{(34)\}$ & $\{(34)\}$ \\
quasiuniversal 2 & $\{(43)\}$ & $\{(43),(46),(53)\}$ \\
quasiuniversal 3 & $\{(34),(43)\}$ & $\{(34),(43),(46),(53)\}$ \\
quasiuniversal 4 & $\{(05)\}$ & $\{(05),(60)\}$ \\
quasiuniversal 5 & $\{(05),(34)\}$ & $\{(05),(34),(60)\}$ \\
quasiuniversal 6 & $\{(05),(43)\}$ & $\{(03),(05),(40),(43),(46),(53),(60)\}$ \\
quasiuniversal 7 & $\{(05),(34),(43)\}$ & $\{(02),(03),(05),(10),(34),(40),(43)$, \\
& & $(46),(53),(60)\}$ \\
\hline
\end{tabular}

TABLE 3. The quasiuniversals.

Proposition 5.4. Let $X$ be a topological space, and let $A \subseteq X$. Let $u$ and $v$ be words. Then

- A has property $C(u)<C(v)$ if and only if $\operatorname{cl}(A)$ has property $u<v$;

- A has property $I(u)<I(v)$ if and only if $\operatorname{int}(A)$ has property $u<v$;

- A has property $d(v)<d(u)$ if and only if $A^{\prime}$ has property $u<v$.

Proof: This follows immediately from Proposition 5.3.

If $U$ is the universal of $X$, then for every $A \subseteq X$, we must have that $\operatorname{cl}(A)$, $\operatorname{int}(A)$, and $A^{\prime}$ have all the properties in $U$. Thus the system $\mathfrak{U}$ should also have rules of inference such as "from $u<v$ infer $C(u)<C(v)$," "from $u<v$ infer $I(u)<I(v)$," and "from $u<v$ infer $d(v)<d(u)$." Let $\mathfrak{U}_{q}$ be the system obtained from $\mathfrak{C l}$ by adding these rules of inference.

Again, we can simplify our discussion by identifying those elementary properties that are "universalelementary," in other words, to identify a set $\mathcal{F}$ such that for every $P \in \mathcal{E}$ there is an $S \subseteq \mathcal{F}$ with $S \vdash_{\mathfrak{U}_{q}} P$ and $P \vdash_{\mathfrak{U}_{q}} Q$ for every $Q \in S$.

Again, we first find the properties that are "universalcanonical," that is, such that $P \preccurlyeq Q$ for every $Q$ with $\operatorname{Con}_{\mathfrak{U}_{q}}(P)=\operatorname{Con}_{\mathfrak{U}_{q}}(Q)$. With a modified version of the script canonical, we find that there are eight universalcanonical properties, namely

$$
\mathcal{C}_{\mathfrak{U}}=\{(00),(02),(03),(04),(05),(34),(43),(56)\} .
$$

We then make the following definition:

Definition 5.5. A property $P$ is universal-elementary if $P \in \mathcal{C}_{\mathfrak{U}}$ and

$$
P \notin \operatorname{Con}_{\mathfrak{U}_{q}}\left(\left(\operatorname{Con}_{\mathfrak{U}_{q}}(\{P\}) \cap \mathcal{C}_{\mathfrak{U}}\right) \backslash\{P\}\right) .
$$

Again, we may find these using a modified version of the script find_elementaries. The universalelementary properties are (05), (34), and (43). Again, a modified version of check_elementary confirms that each property is equivalent in $\mathfrak{U}_{q}$ to a conjunction of properties from $\mathcal{E}_{\mathcal{U}}=\{(05),(34),(43)\}$, so we may use these as our universal-elementary properties.

Definition 5.6. A subset $U$ of $\mathcal{E}$ is a quasiuniversal if it is the intersection with $\mathcal{E}$ of a set that is closed under the rules of inference of $\mathfrak{U}_{q}$, or equivalently if $U=\mathcal{E} \cap$ $\mathrm{Con}_{\mathfrak{U}_{q}}(U)$.

Clearly, if $U$ and $V$ are quasiuniversals, then $U=V$ if and only if $U \cap \mathcal{E}_{\mathcal{U}}=V \cap \mathcal{E}_{\mathcal{U}}$. Thus there are at most $2^{\left|\mathcal{E}_{\mathcal{U}}\right|}=8$ quasiuniversals. We may readily confirm that all of these sets are indeed quasiuniversals, using a modified version of the script types called quasi-universals. They are listed in Table 3.

The script find_types was modified to give the script find_universals described in Section 7.7. This script was used to find examples of quasiuniversals $0,1,2,3,6$, and 7 , but no examples of quasiuniversals 4 and 5 . This is because of the following result.

Lemma 5.7. Let $X$ be a topological space in which every subset is $\beta$-open (i.e., has property (05), that is, A $<$ clint cl A). Then every open set in $X$ is closed.

Proof: Let $U$ be an open set in $X$, and put $A=\partial U=$ $\operatorname{cl}(U) \backslash U$. Then $A$ is $\beta$-open, so $A \subseteq \operatorname{cl}(\operatorname{int}(\operatorname{cl}(A)))$. But int $(\operatorname{cl}(A))=\varnothing$, so $A \subseteq \operatorname{cl}(\varnothing)=\varnothing$. Thus $\partial U=\varnothing$ for every open set $U$; in other words, every open set is closed.

Corollary 5.8. Let $S$ be a universal with $(05) \in S$. Then $(43) \in S$. 


\begin{tabular}{|c|c|c|c|}
\hline & $U \cap \mathcal{E}_{\mathcal{U}}$ & $U$ & Order \\
\hline universal 0 & $\varnothing$ & $\varnothing$ & $a e, a f, b e, c f, e b, h a, h c$ \\
\hline universal 1 & $\{(34)\}$ & $\{(34)\}$ & $e a, e f$ \\
\hline universal 2 & $\{(43)\}$ & $\{(43),(46),(53)\}$ & $b e, d h, f d, h d$ \\
\hline universal 3 & $\{(34),(43)\}$ & $\{(34),(43),(46),(53)\}$ & be \\
\hline universal 4 & $\{(05),(43)\}$ & $\begin{array}{l}\{(03),(05),(40),(43),(46), \\
(53),(60)\}\end{array}$ & $\begin{array}{l}a c, a f, a g, a h, c d, c e, d a, e d, \\
f g, g e, h c, h d\end{array}$ \\
\hline universal 5 & $\{(05),(34),(43)\}$ & $\begin{array}{l}\{(02),(03),(05),(10),(34), \\
(40),(43),(46),(53),(60)\}\end{array}$ & \\
\hline
\end{tabular}

TABLE 4. The universals.

Proof: By the previous lemma, we know that every open set is closed; in other words, we have $\operatorname{cl}(\operatorname{int}(A)) \subseteq \operatorname{int}(A)$ for each $A \subseteq X$, so every subset $A$ of $X$ satisfies cl int $\mathrm{A}<$ int $A$, or (42). Since $e c(\{(42)\})=\{(40),(43),(46),(60)\}$, we have $(43) \in S$, as required.

In light of Corollary 5.8, we obtain the system $\mathfrak{U}$ from $\mathfrak{U}_{q}$ by adding the rule of inference "from (05) infer (43)." Adding this rule of inference to quasi-universals to get the script universals, we find that there are exactly six subsets of $\mathcal{P}$ that are closed under the rules of inference of $\mathfrak{U}$, namely those sets whose intersections with $\mathcal{E}$ are quasiuniversals $0,1,2,3,6$, and 7 .

Theorem 5.9. There are precisely six universals.

Proof: Clearly, every universal is the intersection with $\mathcal{E}$ of a subset of $\mathcal{P}$ that contains the axioms and is closed under the rules of inference of $\mathfrak{U}$. Thus there are at most six universals.

In Table 4 we list examples of topological spaces with each of these six universals. In each case, the underlying set $X$ is $\{a, b, \ldots, h\}$, and the topology is that with specialization order generated by the given edges. For example, the row "universal $1|\{(34)\}|$ ea, ef" means that the topology on $X$ with specialization order generated by $e<a, e<f$ has universal $u(X)=\{(34)\}$.

Note that universal 5 is the property that the topology is discrete, which is why the example has no generators in its specialization order. The other universals have also been studied in the literature.

Definition 5.10. Let $X$ be a topological space.

1. $X$ is extremally disconnected if the closure of every open set is open.
2. $X$ is irresolvable if it cannot be written as a union of two disjoint dense subsets. It is strongly irresolvable (or open hereditarily irresolvable) if every nonempty open subset is irresolvable.

3. $X$ is a partition topology if there is an equivalence relation on $X$ such that a subset is open if and only if it is a union of equivalence classes, or equivalently if every closed set is open.

Theorem 5.11. Let $X$ be a topological space.

1. $X$ is extremally disconnected if and only if it satisfies universal 2.

2. $X$ is strongly irresolvable if and only if it satisfies universal 1.

3. $X$ is a partition topology if and only if it satisfies universal 4.

Proof:

(1) Note that $X$ is extremally disconnected if and only if the closure of $\operatorname{int}(A)$ is open for each $A \subseteq X$, in other words, if and only if every subset of $X$ satisfies cl int $\mathrm{A}<$ int cl int $\mathrm{A}$, or (46). Thus $X$ is extremally disconnected if and only if $\operatorname{Con}_{\mathfrak{U}}((46)) \subseteq u(X)$. Since (46) and (43) are equivalent in $\mathfrak{U}$, a space is extremally disconnected if and only if it satisfies universal 2.

(2) We first observe that if $X$ is strongly irresolvable and $A$ is dense in an open set $U$, then $\operatorname{int}(A)$ is dense in $U$. Otherwise, there would be a nonempty open $V \subseteq$ $U \backslash \operatorname{int}(A)$, and $V$ would be the union of the sets $V \backslash A$ and $V \cap A$, each of which is dense in $V$.

So suppose that $X$ is strongly irresolvable. Let $A \subseteq$ $X$. We must show that $\operatorname{int}(\operatorname{cl}(A)) \subseteq \operatorname{cl}(\operatorname{int}(A))$. So let $B=\operatorname{int}(\operatorname{cl}(A)) \backslash \operatorname{cl}(\operatorname{int}(A))$. Note that $B$ is open and $A \cap B$ is dense in $B$. Thus by the above observation 
$U=\operatorname{int}(A \cap B)$ is dense in $B$. But $U \subseteq \operatorname{int}(A)$ so $B \subseteq$ $\operatorname{cl}(\operatorname{int}(A))$, so $B=\varnothing$, as required.

Conversely, suppose $X$ is not strongly irresolvable. Then there is a nonempty open set $U$ with disjoint subsets $A$ and $B$ that are both dense in $U$. On the one hand, we have $U \subseteq \operatorname{int}(\operatorname{cl}(A))$. On the other hand, $\operatorname{int}(A)$ is open in $U$ and misses $B$, so it is empty. So $\operatorname{int}(\operatorname{cl}(A)) \nsubseteq \mathrm{cl}(\operatorname{int}(A))$, so $A$ does not satisfy (34), so $X$ does not have universal 1.

(3) Suppose first that $X$ is a partition topology. Let $A \subseteq X$. Then $\operatorname{cl}(A)$ is the union of all the equivalence classes meeting $A, \operatorname{socl}(A)$ is open, so we have $A \subseteq$ $\operatorname{cl}(A) \subseteq \operatorname{int}(\operatorname{cl}(A))$. Thus $A$ satisfies (03), as required.

Conversely, suppose $(03) \in u(X)$. If $A$ is closed, then $A \subseteq \operatorname{int}(\operatorname{cl}(A))=\operatorname{int}(A)$, so $A$ is open. Thus every open set is closed, so $X$ is a partition topology.

Another way to view a universal $U$ is to consider the extension $\mathfrak{C} \mathfrak{r}[U]$ of $\mathfrak{C l}$ obtained by adding all the properties in $U$ as axioms.

Theorem 5.12. For each universal $U$, the system $\mathfrak{C r}[U]$ is sound and adequate, in the sense that if $P_{1}, P_{2}, \ldots, P_{n}, Q \in \mathcal{P}$, then $P_{1}, P_{2}, \ldots, P_{n} \vdash_{\mathfrak{C r}[U]} Q$ if and only if for every space $X$ with $u(X) \supseteq U$ and every subset $A$ of $X$ that satisfies $P_{1}, P_{2}, \ldots, P_{n}, A$ satisfies $Q$.

Proof: Soundness again follows from the fact that all the axioms and rules of inference are sound.

For adequacy, we will again consider types. First, consider the subsets of $\mathcal{E}$ that are the intersection with $\mathcal{E}$ of a subset of $\mathcal{P}$ that contains the axioms of $\mathfrak{C}[[U]$ and is closed under the rules of inference of $\mathfrak{C}[U]$. These are precisely the types that contain $U$. There are forty-nine types containing universal 0 , sixteen types containing universal 1 , ten types containing universal 2 , six types containing universal 3 , two types containing universal 4 , and one type containing universal 5 . For each of these universals $U$, examples have been found of spaces with universal $U$ and with each of the appropriate types of subset.

\section{FURTHER DIRECTIONS}

Our system $\mathfrak{C l}$ and its extensions discussed above are adequate for properties of spaces of the form "all subsets have property $P$." We might extend this idea to properties of spaces of the form "each subset with property $P$ has property $Q . "$
Recall that a subset $A$ of a topological space $X$ is nowhere dense if $\operatorname{int}(\operatorname{cl}(A))=\varnothing$. Van Douwen introduced the class of nodec spaces: those spaces in which every nowhere dense set is closed [van Douwen 93]. This class is important, for example, in the study of submaximal spaces. Although superficially not being of the form mentioned above (since the definition involves a "constant" $\varnothing)$, Cao, Greenwood, and Reilly have shown that a space is nodec if and only if every $\alpha$-closed subset is closed [Cao et al. 01, Theorem 2.10], in other words, if every subset with property (50) has property (10). Since we have

$$
(50) \Leftrightarrow(34) \wedge(40) \wedge(60),
$$

we might abbreviate this as $(\forall A)((34) \wedge(40) \wedge(60) \Rightarrow$ (10)).

If we inspect the list of types in Table 1, we see that only Types 28 and 46 violate this: thus a space is nodec if and only if it has no subsets of Type 28 or 46 . Of course, the dual property is also true: all $\alpha$-open sets are open, so Types 5 and 18 are similarly excluded. Once again, a modified version of $f$ ind_types has found examples of all 45 types of subsets of nodec spaces.

Again, we can think of the nodec property as being an extension of $\mathfrak{C l}$ or $\mathfrak{C l}_{-}$, this time obtained by adding the new rules of inference "from (34) and (40) and (60) infer (10)" and "from (03) and (05) and (34) infer (02)," and the same argument as before shows that this system is sound and adequate for nodec spaces.

Another direction for future research is to consider a slightly richer language, for example, adding constant symbols for $\varnothing$ and $X$, or a binary operation symbol for $\checkmark$. Note that if we introduce both a constant symbol for $X$ and a binary operation symbol for $\backslash$, we will in effect have complements, unions, and interiors. In general, these three operations can generate infinitely many distinct sets [Bowron and Rabinowitz 97].

\section{PROGRAMS}

The programs used to develop the results presented in this paper were all written in the scripting language Perl. They are described briefly in the following subsections. The scripts themselves are available for download at http: //www.math.auckland.ac.nz/ mcintyre/ptc.

The following conventions are used:

- Properties are represented by two-digit strings such as 02 .

- The operations $c, i, d, C$, and $I$ on $W$ are represented by arrays @c, @i, etc. For example, $\$ c[2]==4$, 
because $c($ int $\mathrm{A})=\mathrm{cl}$ int $\mathrm{A}$, and we number int $\mathrm{A}$ as 2 and $c l$ int $A$ as 4 .

- When finding $\operatorname{Con}(S)$, we usually use an associative array (or hash) \%hold, with \$hold $\{$ uv\} == 1 if $(u v) \in \operatorname{Con}(S)$, and \$hold $\{u v\}$ undefined otherwise. This means that we can extract $\operatorname{Con}(S)$ using keys \%hold.

\subsection{The Script full_cons}

The main part of the script is the subroutine find_consequences, which calculates $\operatorname{Con}_{\mathfrak{C r}}(S)$. This first sets $\$$ hold $\{\mathrm{P}\}=1$ for each axiom $P$ and each $P \in S$. It then enters a loop: in each iteration, we apply each of the rules of inference to each property known to hold. We check the number of properties known to hold at the start and end of each loop. When these values are equal, we exit the loop.

\subsection{The Script canonical}

The script canonical finds the canonical properties. Recall that these are defined as those properties $P$ such that $P \preccurlyeq Q$ whenever $P \Leftrightarrow Q$, and note that $P \Leftrightarrow Q$ if and only if $\operatorname{Con}_{\mathfrak{C r}}(P)=\operatorname{Con}_{\mathfrak{C r}}(Q)$. The script works as follows. We consider the properties in lexicographical order. For each property $P, S=\operatorname{Con}_{\mathfrak{C r}}(\{P\})$ is found using the subroutine find_consequences described above. We then check the associative array found_canonical to see whether found_canonical $\{\mathrm{S}\}$ is defined. If not, then we declare $P$ to be canonical and set found_canonical $\{\mathrm{S}\}$ to be true.

\subsection{The Script find_elementaries}

The script find_elementaries first uses the above method to identify the canonical properties. It then runs through all the canonical properties $P$ : for each $P$ it finds the consequences of $P$, finds those consequences that are canonical and distinct from $P$, finds the consequences of this list, and checks whether $P$ is in this latter set of consequences. If not, $P$ is elementary.

\subsection{The Script check_elementary}

This script takes as input a list $E$ of properties and tests whether each property is equivalent to a conjunction of properties in $E$. For each $P$, it first uses find_consequences to find $\operatorname{Con}_{\mathfrak{C r}}(\{P\})$, then intersects this with $E$ using the subroutine get_elementaries. It then finds the consequences of this set, and checks that the original property $P$ is in this list.

\subsection{The Script cl-minus}

This script takes each $S \subseteq \mathcal{E}$, finds $\operatorname{Con}_{\mathfrak{C r}}(S)$ using find_consequences, and intersects it with $E$. It also finds the consequences of $S$ in the restricted system $\mathrm{Cl}_{-}$, using the subroutine restricted_consequences. This latter subroutine is similar in structure to find_consequences. However, note that the rules of inference are now concrete instances rather than rule schemata.

\subsection{The Scripts types and find_types}

The script types finds the types. It successively considers each subset of the list of elementary properties, and tests whether that subset is closed under each of the rules of inference of $\mathfrak{C l}_{-}$.

The script find_types first performs the same code as types in order to identify by number the 49 types. It then generates a number of topologies on a given set, finds each subset of the set, determines the type of that subset, and (if it is a type not previously encountered or has fewer elements than the example previously found) stores the example in an associative array indexed by the type.

The underlying set for the space is stored as the array Qpoints. The topologies are generated as follows. First, a list of all pairs $x y$ with $x \neq y$ is formed, and stored as Qedges. Then, for each edge, a random number is generated between 0 and 100: if this random number exceeds a certain threshold (the "edge probability"), the edge is added to a list of generators. The specialization order for the topology is then the reflexive, transitive closure of the set of generators. This process is repeated a number of times. The edge probability is increased, with a certain number of topologies generated for each edge probability. The range of probabilities, step size, and number of topologies generated are all parameters that can be set by the user.

The method used to determine the type of each subset is as follows. First, for each point $x$, the smallest neigborhood of $m(x)$ is found. This is $\{y \mid x \leq y\}$. Then, given a set $A$, we can find $\operatorname{int}(A)$ as $\{y \mid m(y) \subseteq A\}$ and $\operatorname{cl}(A)$ as $\{y \mid m(y) \cap A \neq \varnothing\}$. Now for each set $A$, the sets $w_{A}$ for each $w \in W$ are found, and for each elementary property $w<v$, we test whether $w_{A} \subseteq v_{A}$.

\subsection{The Scripts quasi-universals, universals, and find_universals}

The script quasi-universals is a modification of the script types. 
Conceptually it is obtained by adding all the rules of inference of the form "from $w<v$ infer $C(w)<C(v)$," and similarly for the operators $I$ and $d$. However, in most cases $C(w)<C(v)$ and $I(w)<I(v)$ are not elementary even when $w<v$ is. Thus in fact, the extra rules are all rules of the form "from $w<v$ infer $w^{\prime}<v^{\prime}$," where $w^{\prime}<v^{\prime} \in \operatorname{Con}_{\mathfrak{C r}}(C(w)<C(v)) \cap \mathcal{E}$, and similarly for $I$ and $d$. The script universals is obtained from quasi-universals by adding the rule "from (05) infer (43)" from Corollary 5.8.

The script find_universals is a modified version of the script find_types. A number of topologies are generated in the same way. Once again, the type of each subset is found, giving a list of types found in this topology. Then each elementary property is tested to check whether it occurs in each type found in this topology.

In this way, the universal for this topology is found. The script also records which types of subset have been seen in a space with each given universal: as mentioned in Section 5, this may be used to establish the adequacy of the systems $\mathfrak{C r}[U]$.

\section{REFERENCES}

[Bowron and Rabinowitz 97] M. Bowron and S. Rabinowitz. "Problem 10577." American Mathematical Monthly 104 (1997), 169.

[Cao et al. 01] J. Cao, S. Greenwood, and I. Reilly. "Generalized Closed Sets: A Unified Approach." Applied General Topology 2 (2001), 179-189.

[van Douwen 93] E. K. van Douwen. Applications of Maximal Topologies." Topology and Its Applications 51 (1993), 125-139.

[Engelking 89] R. Engelking. General Topology, 2nd ed. Berlin: Heldermann Verlag, 1989.

[Isomichi 71] Y. Isomichi. "New Concepts in the Theory of Topological Space: Supercondensed Set, Subcondensed Set and Condensed Set." Pacific Journal of Mathematics 38 (1971), 657-668.

[Kuratowski 22] C. Kuratowski. "Sur l'operation $A$ de l'analysis situs." Fundamenta Mathematicae 3 (1922), 182-199.

A. E. McCluskey, Department of Mathematics, National University of Ireland, Galway, University Road, Galway, Ireland (aisling.mccluskey@nuigalway.ie)

D. W. McIntyre, Orion Health, PO Box 8273, Symonds Street, Auckland 1150, New Zealand

W. S. Watson, Department of Mathematics, York University, North York, Ontario M3J 1P3, Canada

(watson@watson.math.yorku.ca)

Received June 16, 2005; accepted in revised form December 12, 2006. 